\title{
Using the Baltic Sea to advance algorithms to extract altimetry-derived sea- level data from complex coastal areas, featuring seasonal sea-ice
}

Marcello Passaro ${ }^{1}$, Felix L. Müller ${ }^{1}$, Adili Abulaitijiang², Ole B. Andersen², Denise Dettmering ${ }^{1}$, Jacob L. Høyer ${ }^{3}$, Milla Johansson ${ }^{4}$, Kristine Skovgaard Madsen $^{3}$, Laura Rautiainen ${ }^{4}$, Ida M. Ringgaard ${ }^{3}$, Eero Rinne ${ }^{4}$, Jani Särkkä ${ }^{4}$, Rory Scarrott ${ }^{5}$, Christian Schwatke ${ }^{1}$, Florian Seitz ${ }^{1}$, Laura Tuomi ${ }^{4}$, Americo Ambrozio $^{6^{*}}$, Marco Restano $6^{6^{* *}}$, Jérôme Benveniste ${ }^{6}$

${ }^{1}$ Deutsches Geodätisches Forschungsinstitut, Technische Universität München (DGFI-TUM), Germany

2 SPACE National Space Institute, Technical University of Denmark, (DTU), Denmark

${ }^{3}$ Danish Meteorological Institute (DMI), Denmark

${ }^{4}$ Finnish Meteorological Institute (FMI), Finland

${ }^{5}$ MaREI Centre, Environmental Research Institute, University College Cork (UCC), Ireland

$6^{*}$ DEIMOS, c/o ESA-ESRIN, Italy

$6^{* *}$ SERCO, c/o ESA-ESRIN, Italy

${ }^{6}$ ESA-ESRIN, Italy
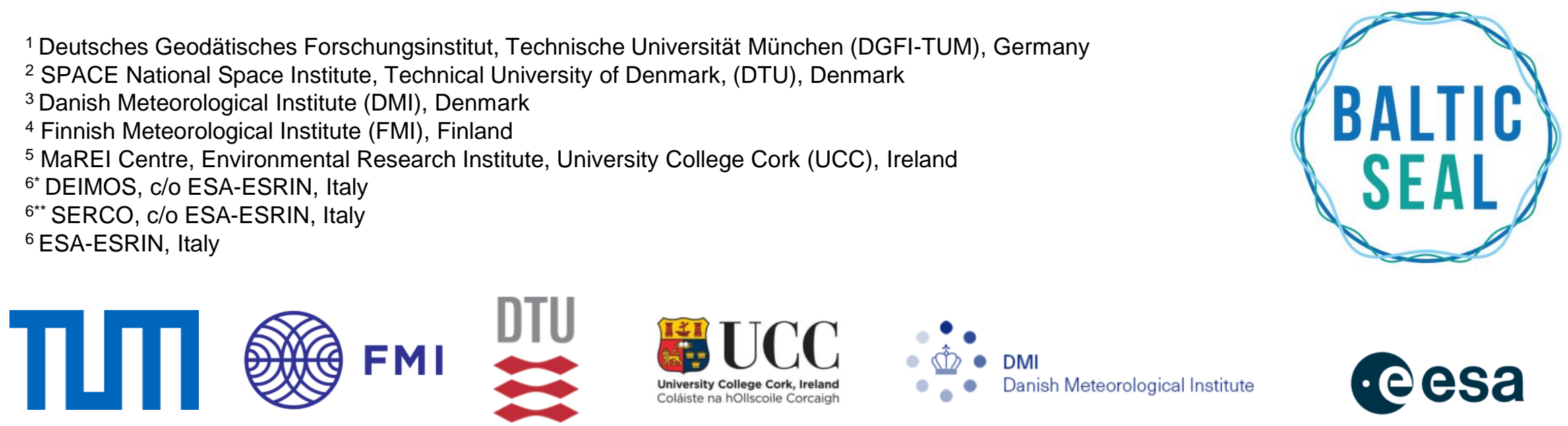

EGU General Assembly 2020

Virtual Presentation, 03.05.2020 


\section{The Baltic Sea - Motivation}

\section{What?}

- Generation of novel long-term multi-mission along-track and gridded sea level products

\section{Why?}

- Previous products show only sparse information in the northern Baltic Sea (no sea-ice treatment)

- Improvements in retracking solutions (closer to the coast), geophysical corrections etc.

- Perfect playground as laboratory for Coastal Altimetry (challenging coastlines and sea-ice coverage)

- Exploiting Artificial Intelligence Algorithms (Unsupervised Radar Waveform Classification, Clustering, K-Medoids)

- Good validation possibilities (large number of tide gauges, optical and SAR image comparisons etc.)

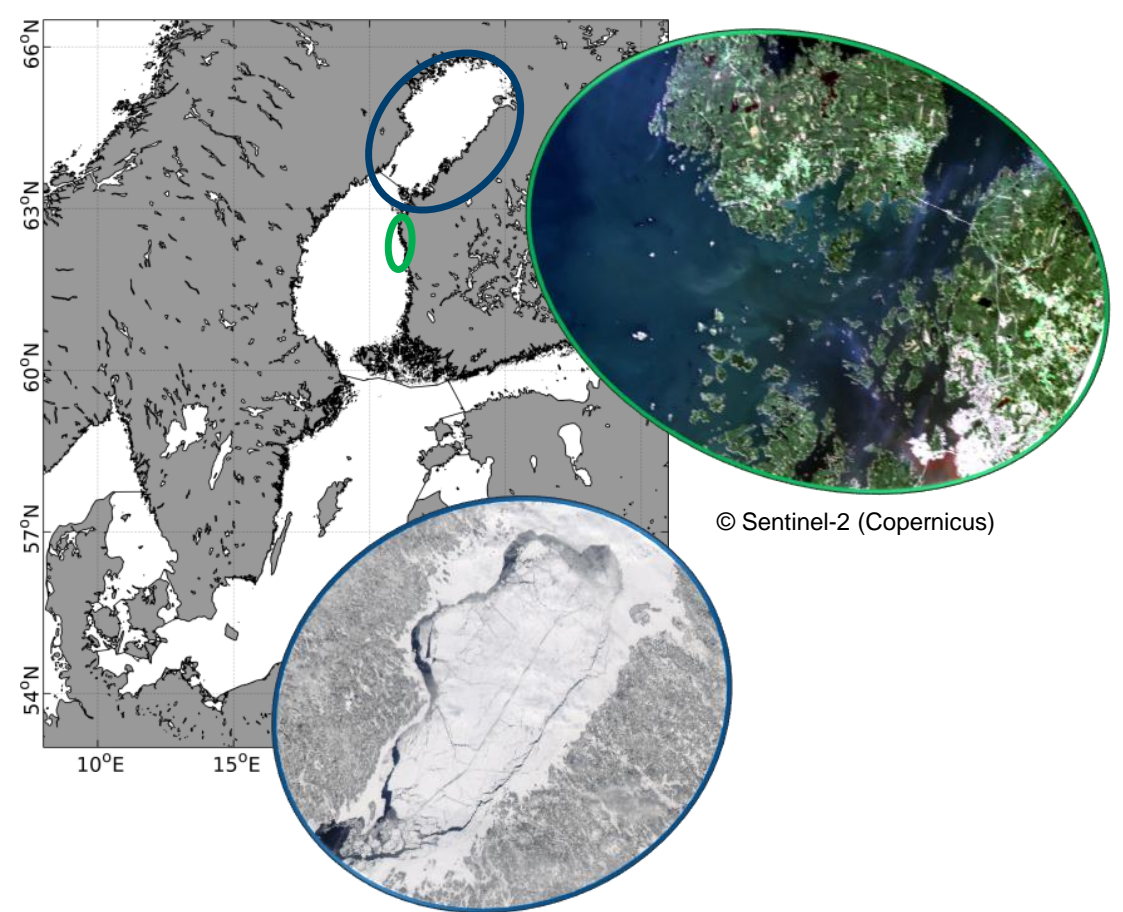

(C) Earth Observing System Data and Information System (EOSDIS), National Aeronautics and Space Administration (NASA)

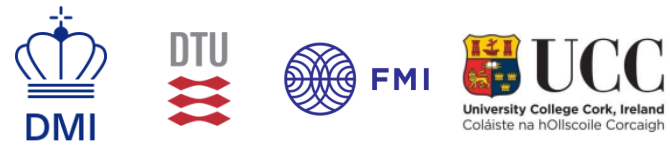




\section{Workflow Baltic+ SEAL}

18 months ESA Tender Project

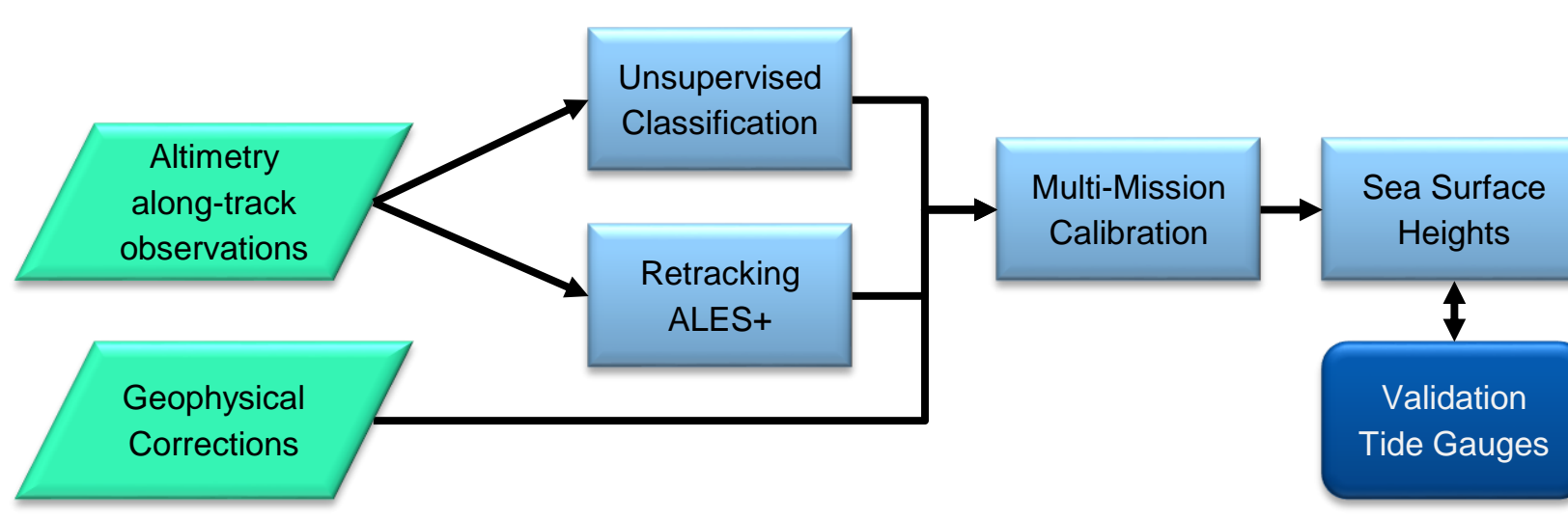

6 Work Packages

1. Scientific Requirements (FMI)

2. Dataset collection (DMI)

3. Algorithm Development and Validation (TUM)

4. Dataset Generation and Impact Assessment (DTU)

5. Scientific Roadmap (UCC)

6. Management and Promotion (TUM) 


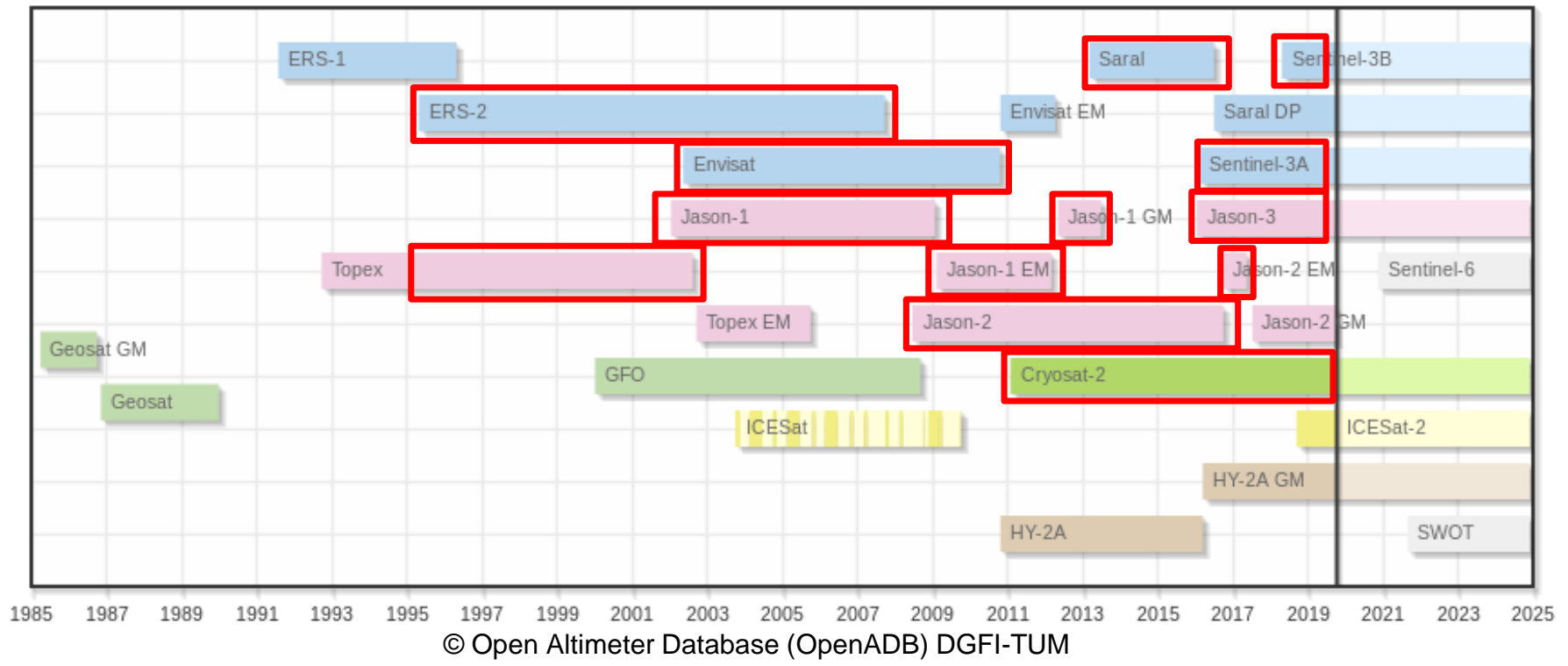

- $\quad 25$ years Multi-mission altimetry data (LRM \& SAR)

- Usage of ALES+ retracked high-frequency along-track observations

- Multi-mission cross calibrated Sea Surface Heights

- Regional cross calibration based on high-frequency along-track observations 


\section{Algorithm Development and Validation - Unsupervised Classification}

- Finding open water (lead) to estimate sea surface heights with multi-mission altimetry data in the northern Baltic Sea region within the sea-ice layer

- Unsupervised classification of waveforms without the use of selected training data for pulse-limited and DelayDoppler altimetry data

- Comparison of classification using optical images and side-looking SAR

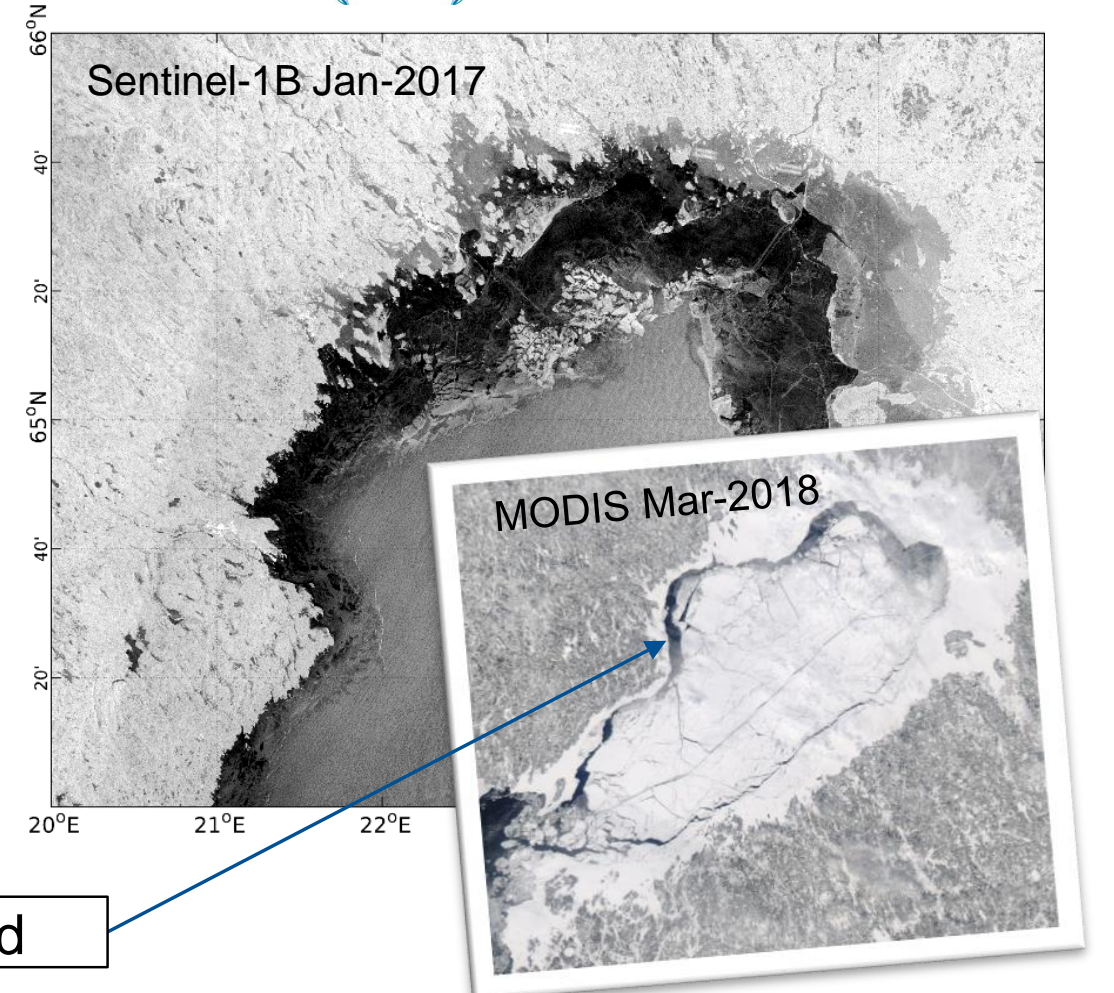

Lead

(0) Earth Observing System Data and Information Syste (EOSDIS), National terion (NASA) 
- Altimeter waveforms (i.e. radar echoes) are affected by surface conditions (e.g. roughness)

- Lead or calm water returns (no wind or waves): single-peak shape, specular behavior, strong backscatter

- Open Ocean waveforms: Brown-like shape, weak backscatter

- Sea-Ice returns: more random shape, backscatter depended on sea-ice surface, strong noise

OCEAN

ERS-2
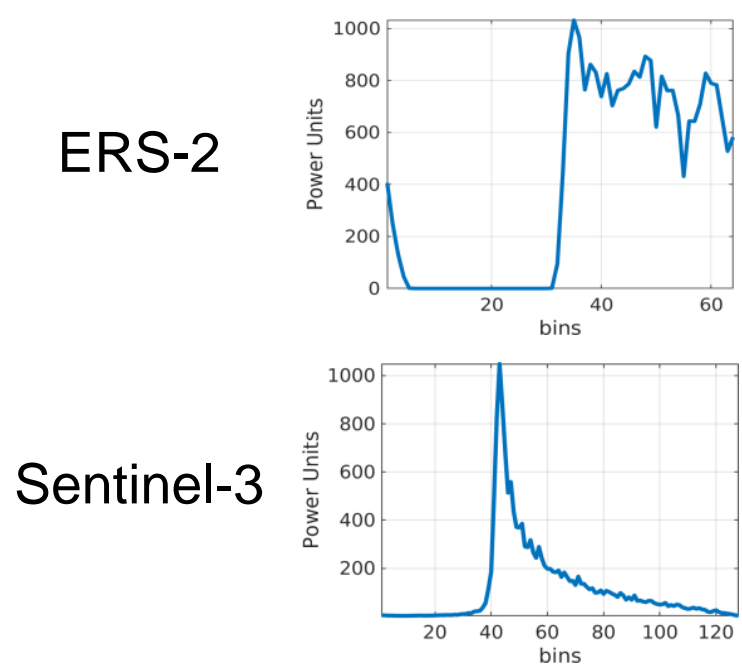

LEAD
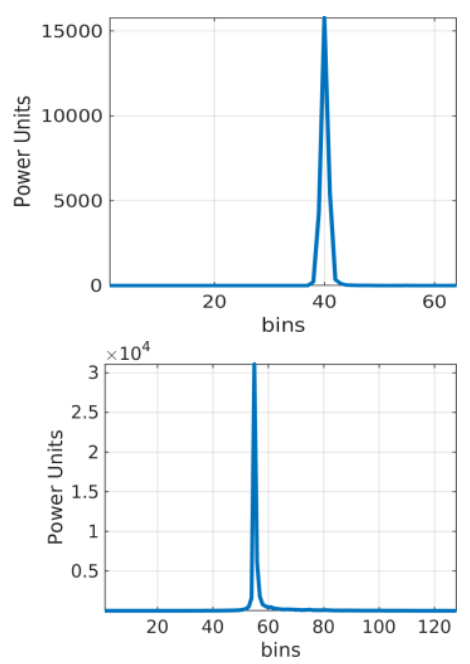
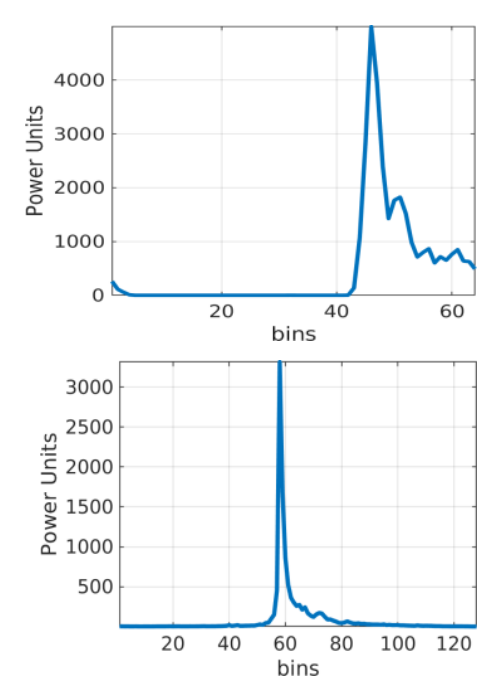

Pulse-Limited

Delay-Doppler

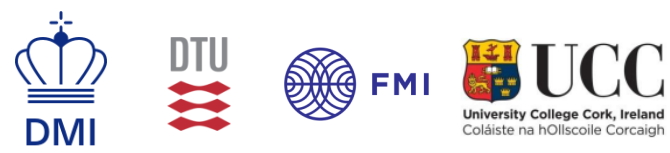




\section{Algorithm Development and Validation - Unsupervised Classification}

- Usage of Artificial Intelligence Algorithms (Data-Mining)

- Application of unsupervised classification

- Input: Original waveform data

- Definition and computation of waveform features

$>$ Maximum Power, waveform width, decay of trailing edge etc. (Parameters describe the waveform's shape and its features)

- Clustering of waveforms in clusters applying K-medoids $>$ Waveform reference model

- Assigning waveform clusters to surface conditions $>4$ classes: calm water, ocean, sea-ice and undefined

- Classification of remaining waveforms using reference model and K-nearest neighbor (K-NN)

- Classification output: WATER [1] | ICE [0] | UNDEFINED [0] (per measurement)

- Same method for LRM and SAR missions, but slightly different feature space

More info: Müller F.L et al.: Monitoring the Arctic Seas: How Satellite Altimetry Can Be Used to Detect Open Water in Sea-Ice Regions. Remote Sensing, 9(6), 551, 10.3390/rs9060551, 2017c

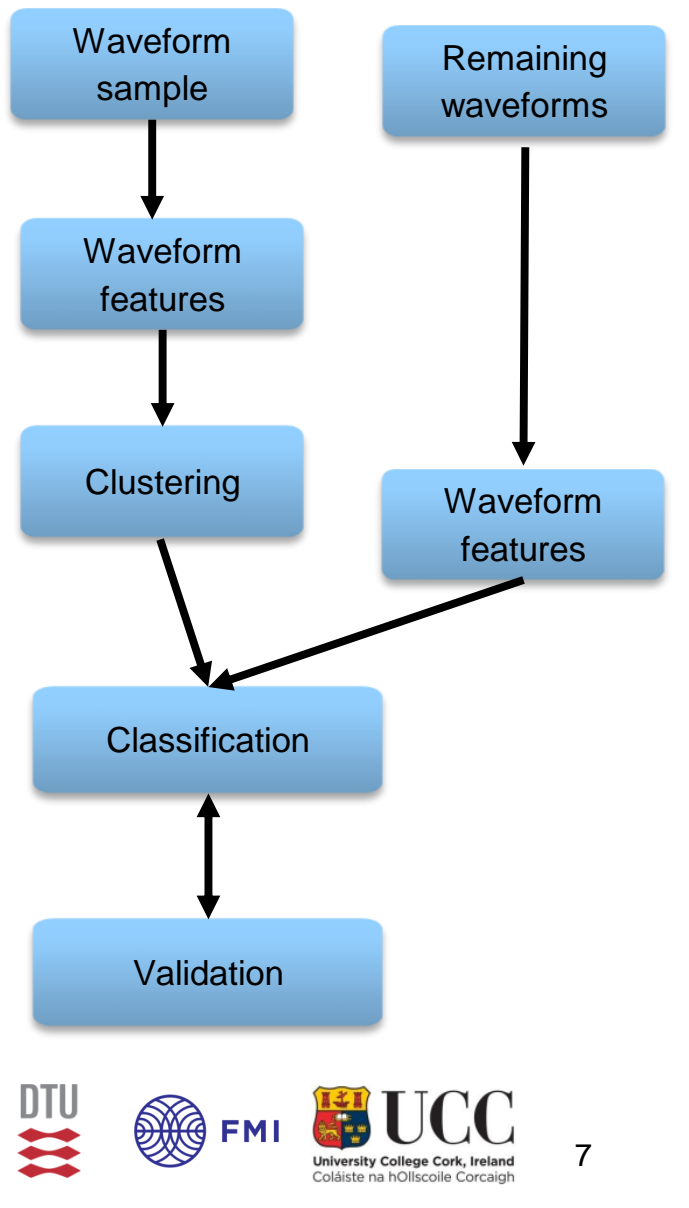




\section{Unsupervised Classification to detect, and exploit leads}

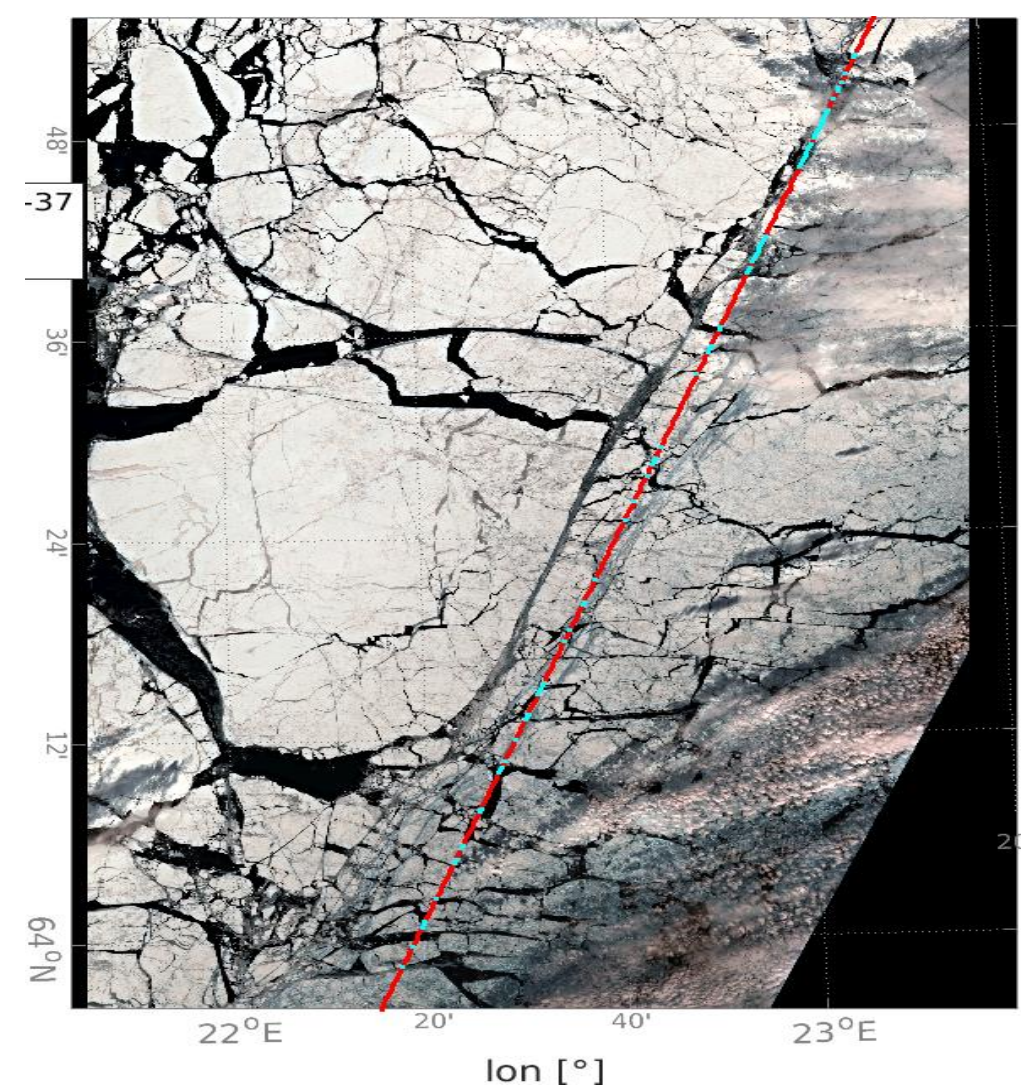

Sentinel-3A vs. Sentinel-2B 2018-04-17 / $\Delta t=37 \mathrm{~min}$

Deutsches Geodätisches Forschungsinstitut (DGFI-TUM) | Technische Universität München (ix)

- Water

- Ice

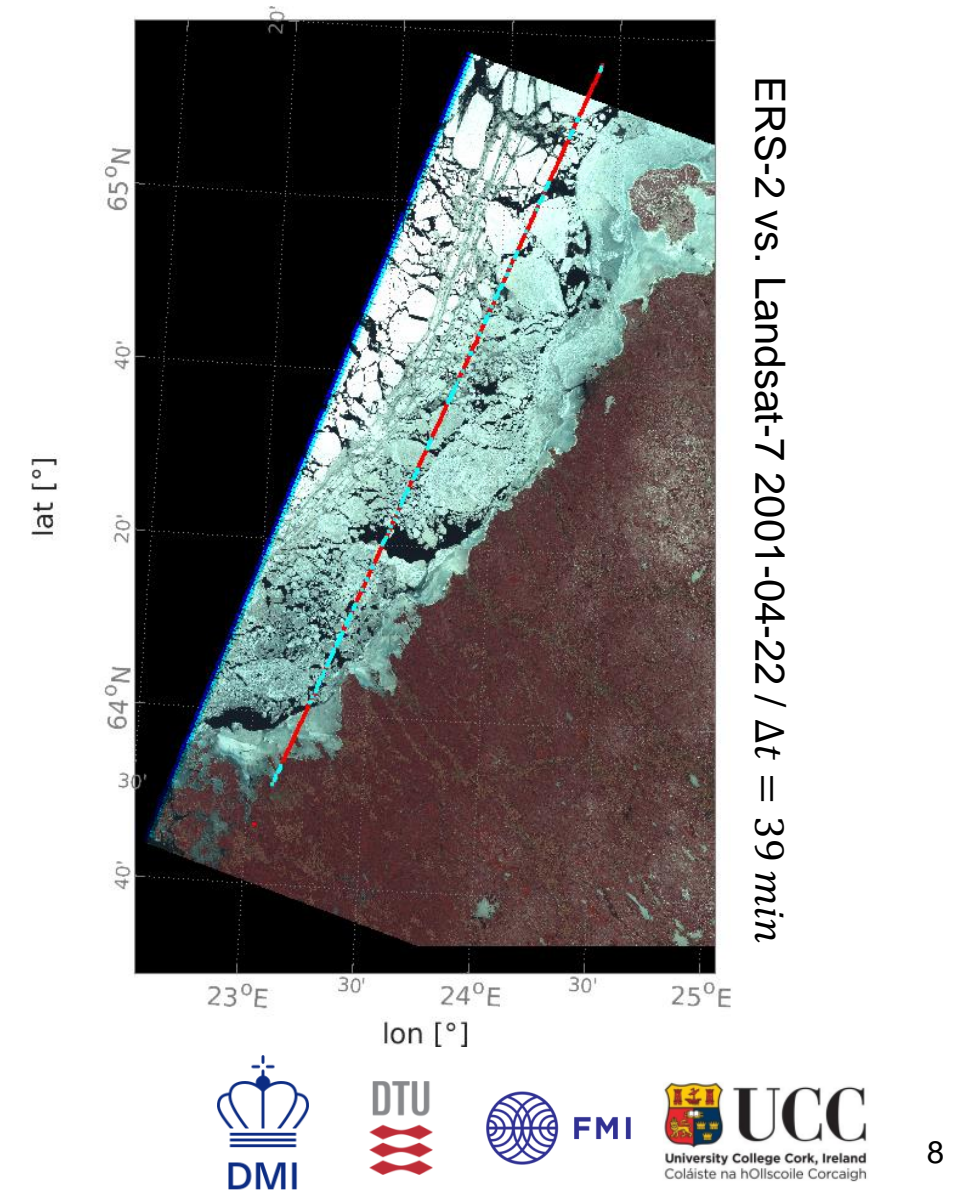




\section{Algorithm Development and Validation (Retracking - ALES+)}

- Sub-waveform retracker to avoid coastal contamination

- Adaptive trailing edge decay to retrack peaky waveforms from leads

- Homogenous range estimation of lead/polynya, open ocean and coastal waveforms (avoids internal biases)

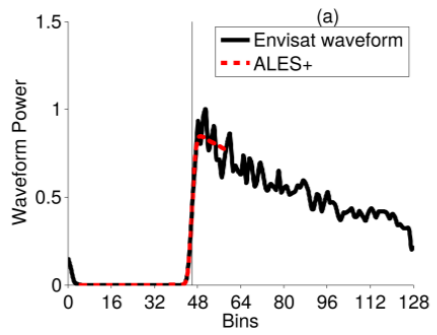

Ocean - LRM

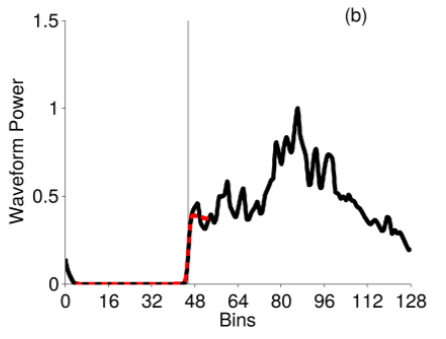

Coastal - LRM

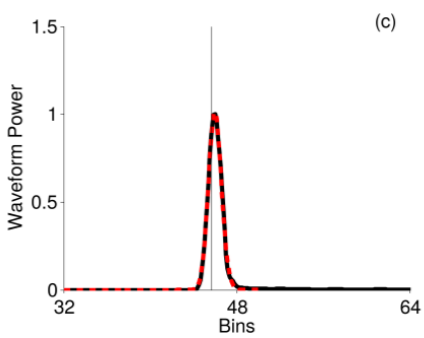

Lead

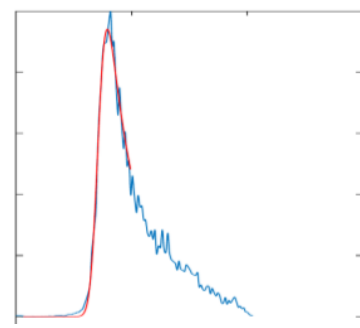

Ocean - SAR

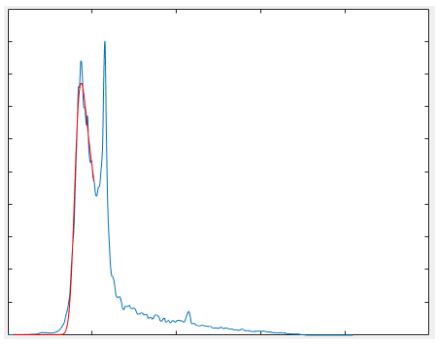

Coastal - SAR

More Information: Passaro M. et al. (2017): ALES+: Adapting a homogenous ocean retracker for satellite altimetry to sea ice leads, coastal and inland waters., Remote Sensing of Environment 


\section{Algorithm Development and Validation (Multi-Mission Cross Calibrationf EAL)}

- Offset not always time-constant $=>$ drifts!

- Differences in sea level heights can have large-scale geographical pattern

- A location-dependent multi-mission cross-calibration (MMXO) between all missions is needed

- Output: time series of radial errors => applied as corrections to each measurement
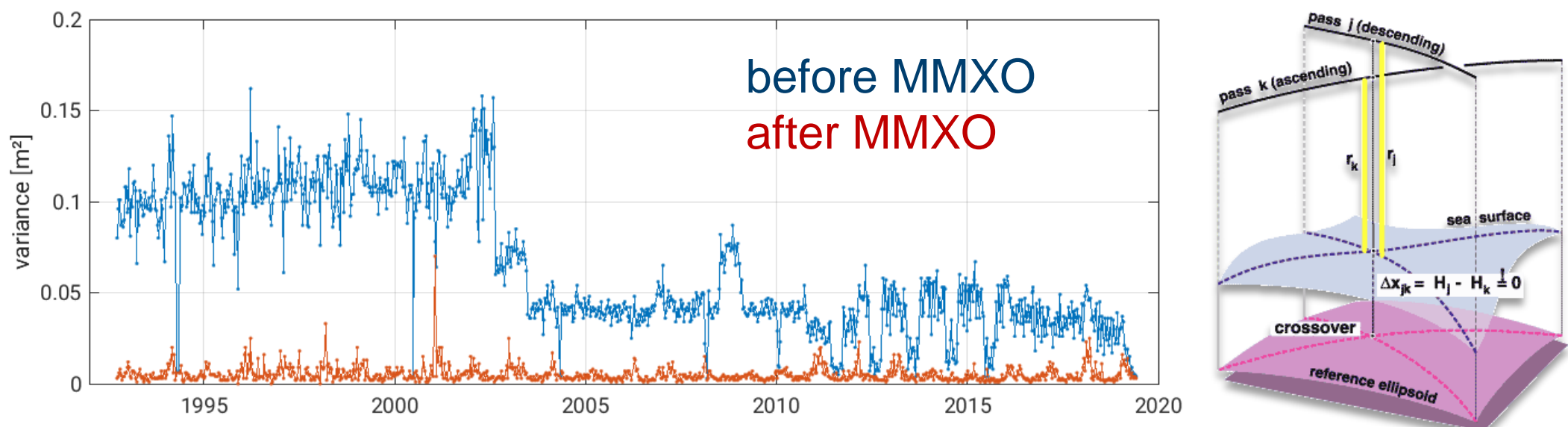

More Information: Bosch W. et al:: Multi-mission cross-calibration of satellite altimeters: constructing a long-term data record for global and regional sea level change studies. Remote Sensing 6(3): 2255-2281, 10.3390/rs6032255, 2014 


\section{Algorithm Development and Validation (Multi-Mission Cross Calibration)ALTIC)

- Multi-mission crossover analysis: regional approach based on high-frequent SSH observations

- Two - four missions per 10 day cycle / max. time differences $=3$ days

\section{Crossover Differences}

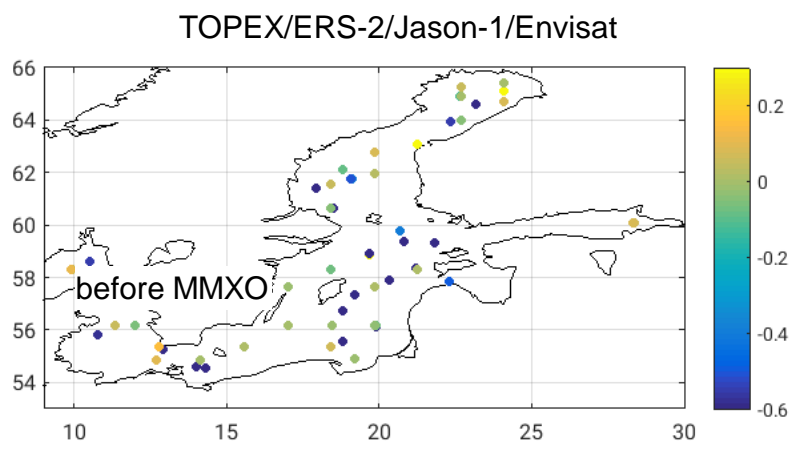

Jason-2/Jason-1 EM/Envisat/Cryosat
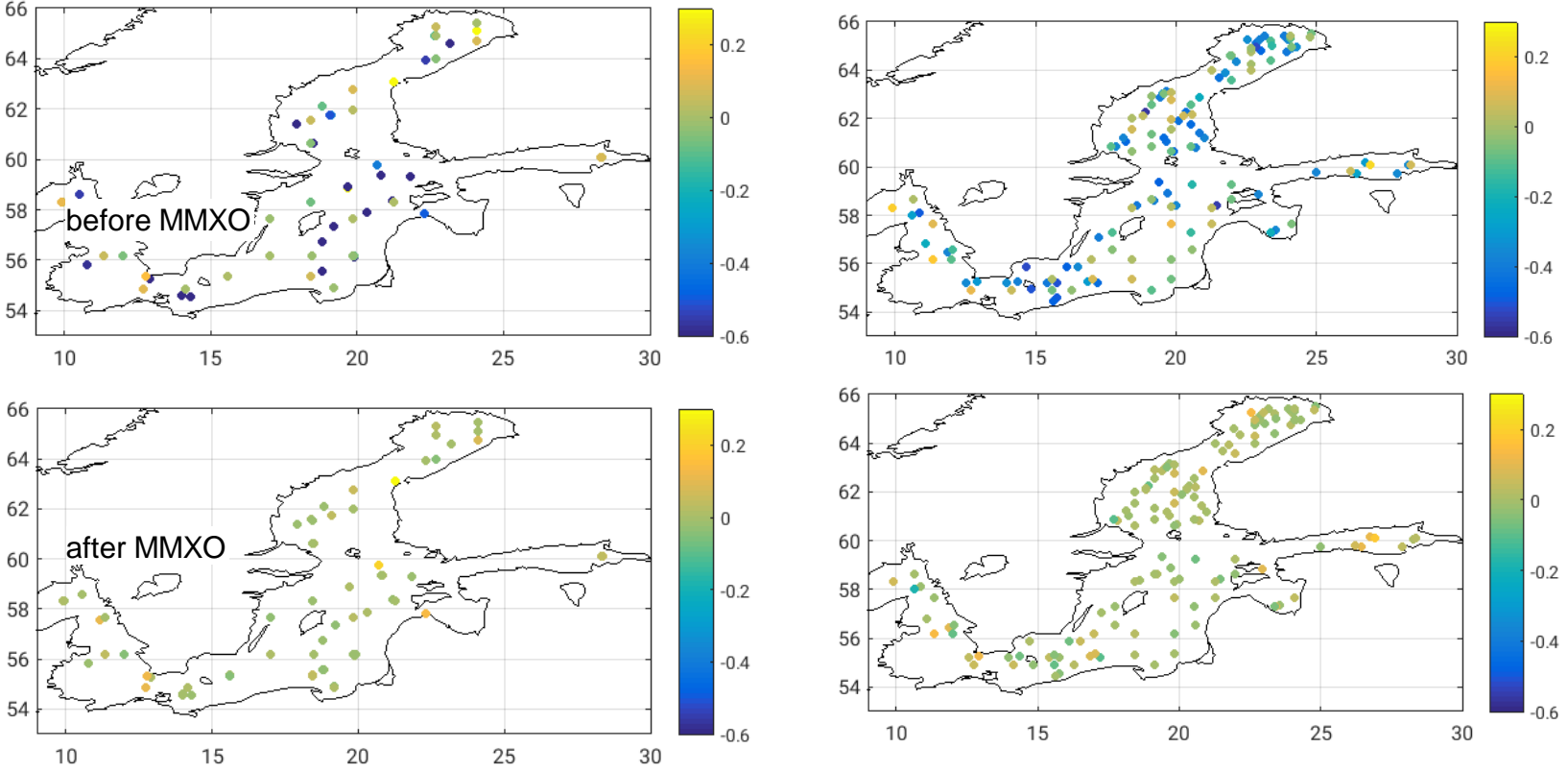

Mean range biases wrt TOPEX

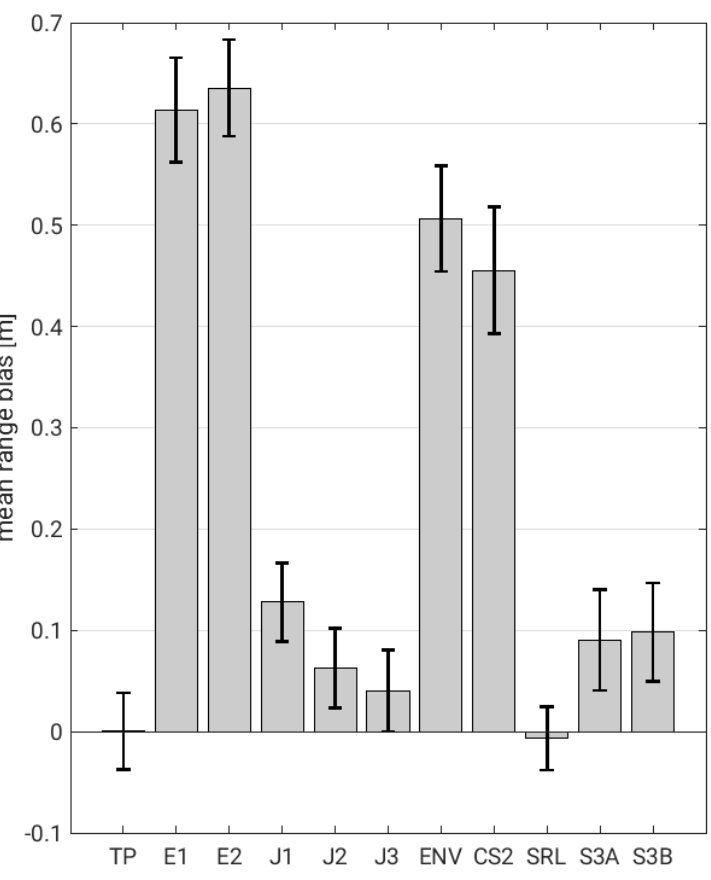




\section{Algorithm Development and Validation (Sea Surface Heights)}

\section{Cesa Tा}

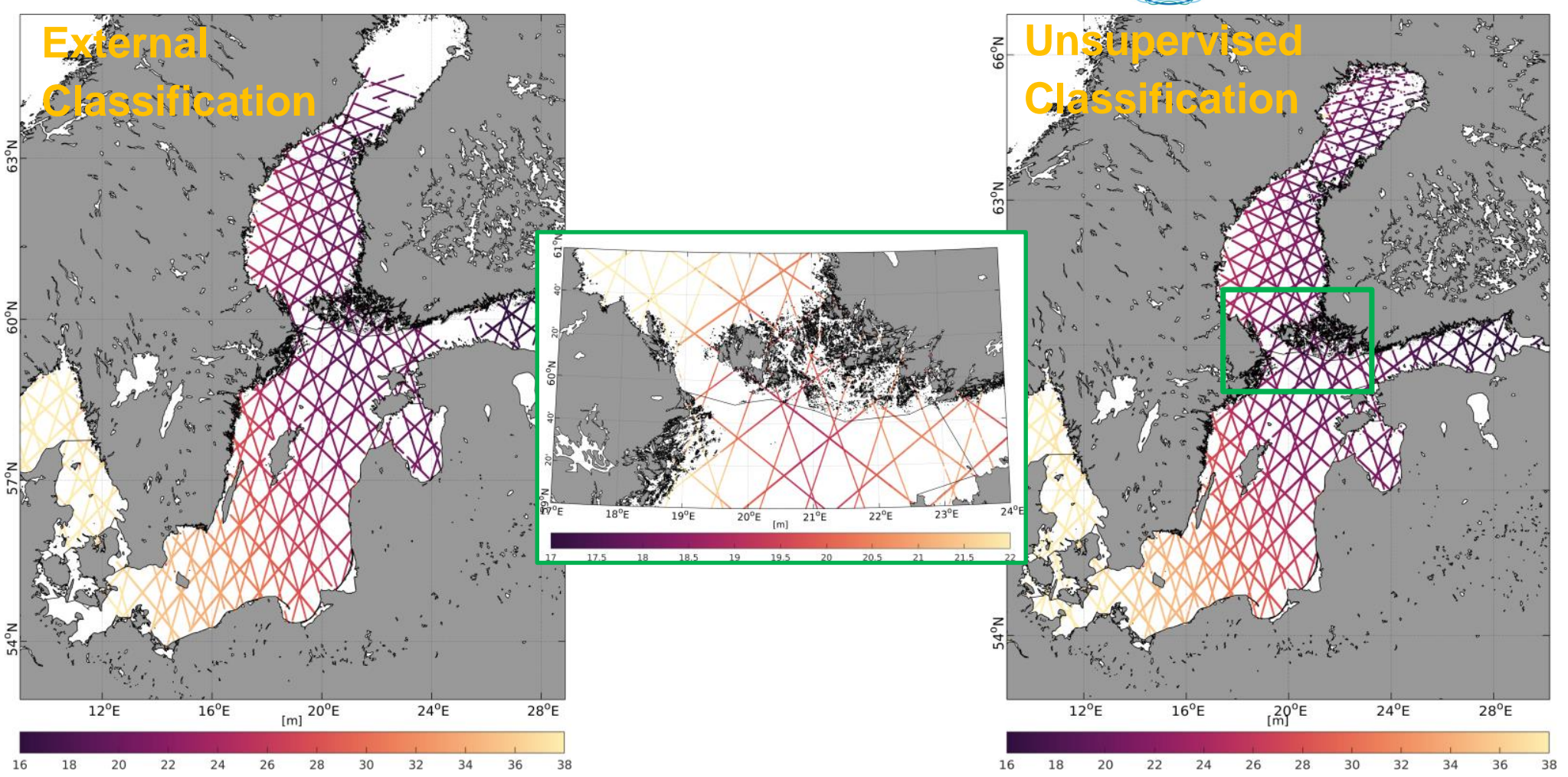

Example: Sea Surface Heights ALES+ 2009-04 (Jason1-EM,Jason-

2,Envisat)

Deutsches Geodätisches Forschungsinstitut (DGFI-TUM) | Technische Universität München

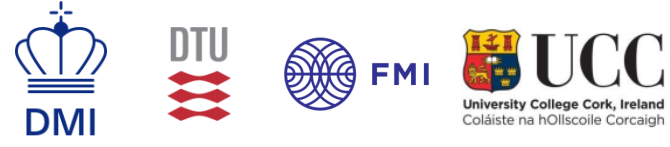




\section{Preliminary Results: Mean Sea Surface}

- A new Mean Sea Surface is being produced within the project

- In the current version, leads among sea ice and SAR altimetry not yet included

- Regional differences between DTU15 and new Mean Surface exist particularly in coastal regions

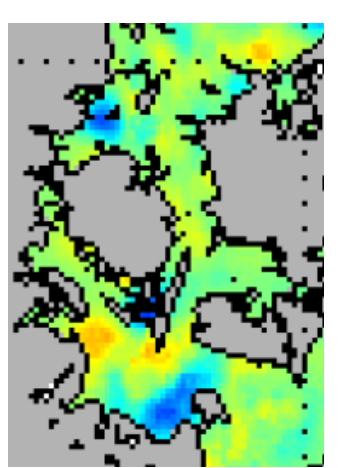

Danish Straits

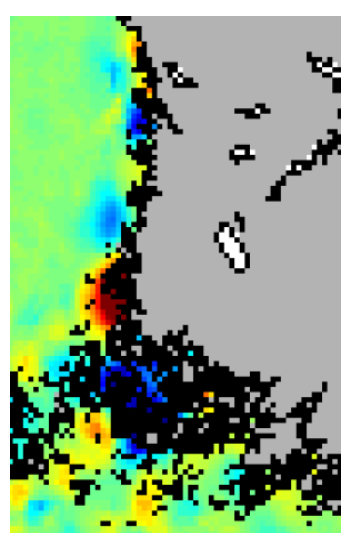

Finnish Coast
Cesa

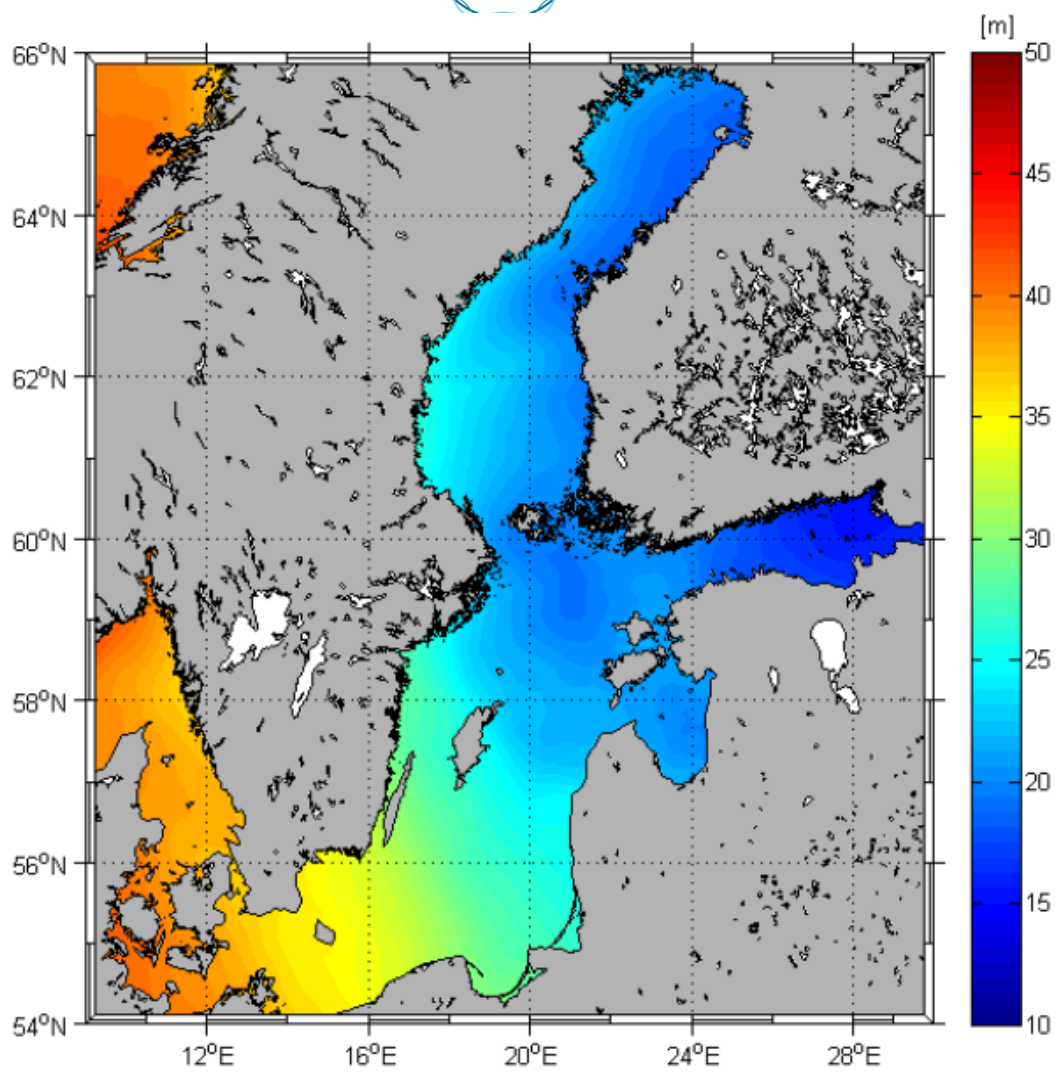

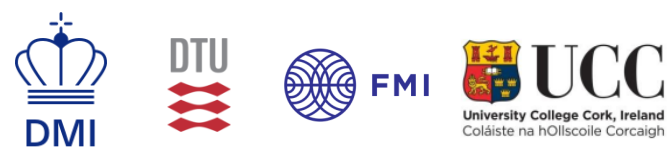


- Gridding along-track SSH data (1995-05 $\rightarrow$ 2019-05) on a triangular, unstructured grid

- Using Least-Squares Methods by fitting an inclined plane to non-regular distributed grid nodes

- Observations are distance-based Gaussian weighted

- Nearly equally spaced grid nodes with 7-8 km spatial resolution
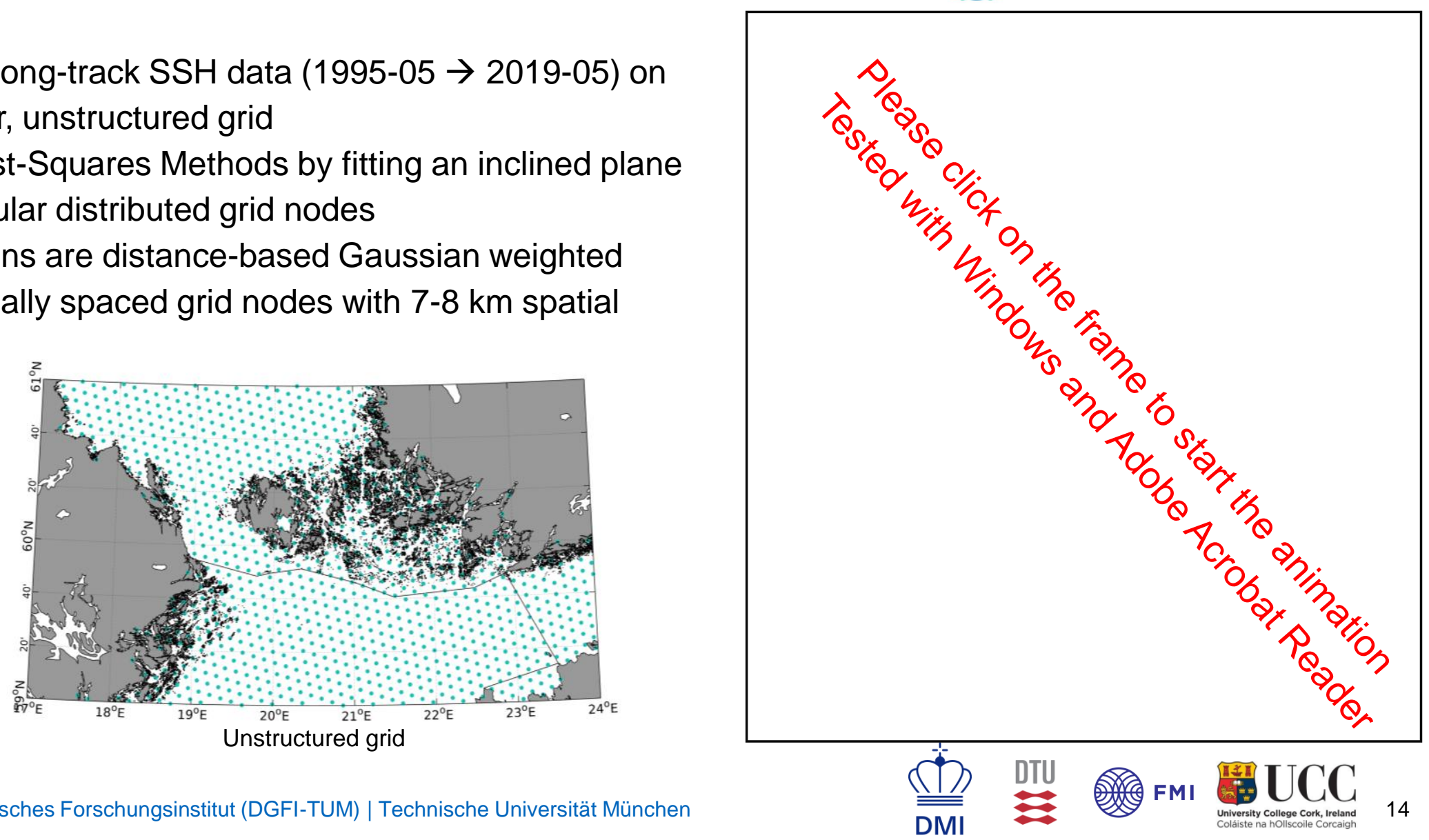
Preliminary Results: Monthly Sea Surface Heights

Cesa ता

- Example of gridded Sea Level Anomalies and uncertainties (2017 March)
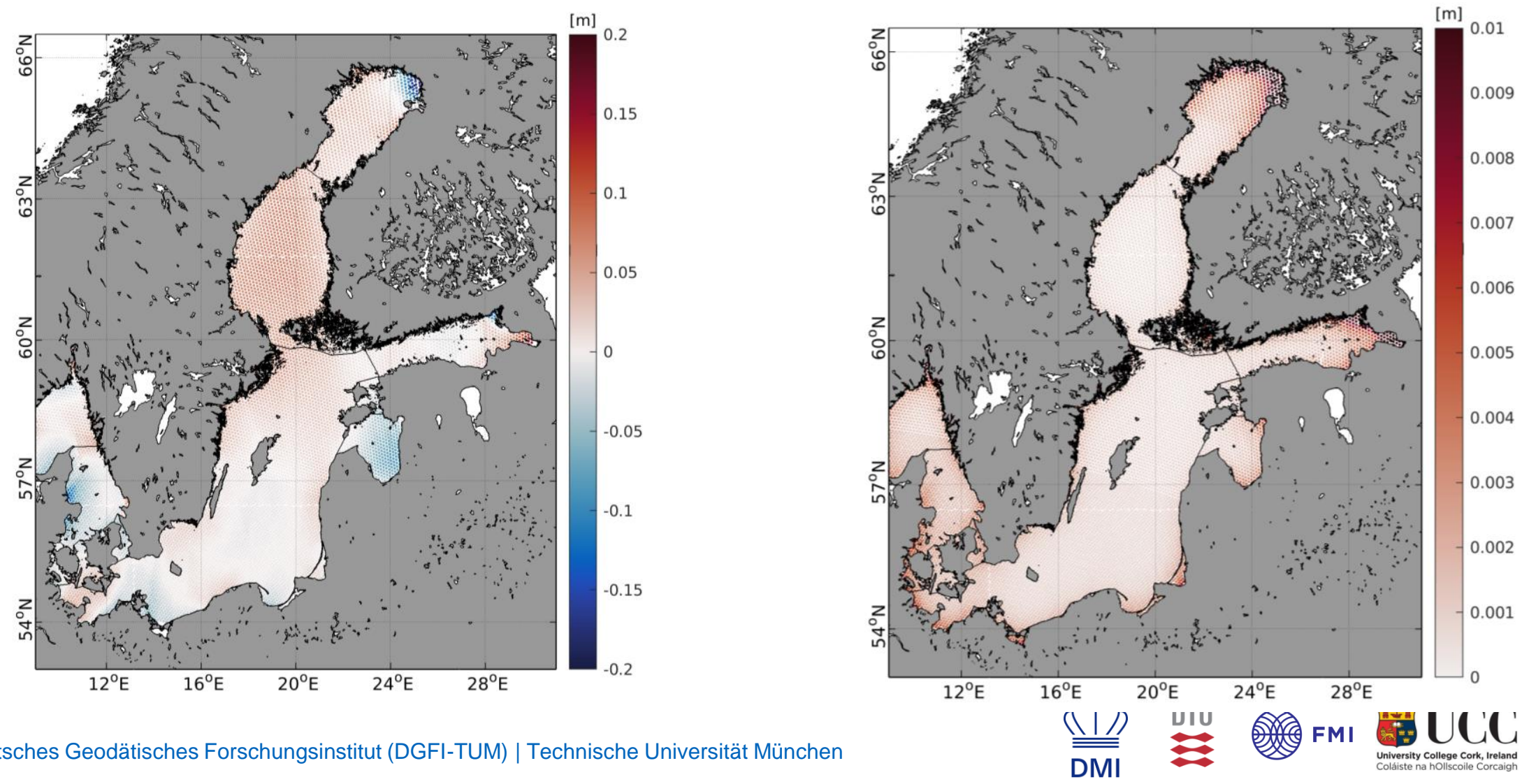


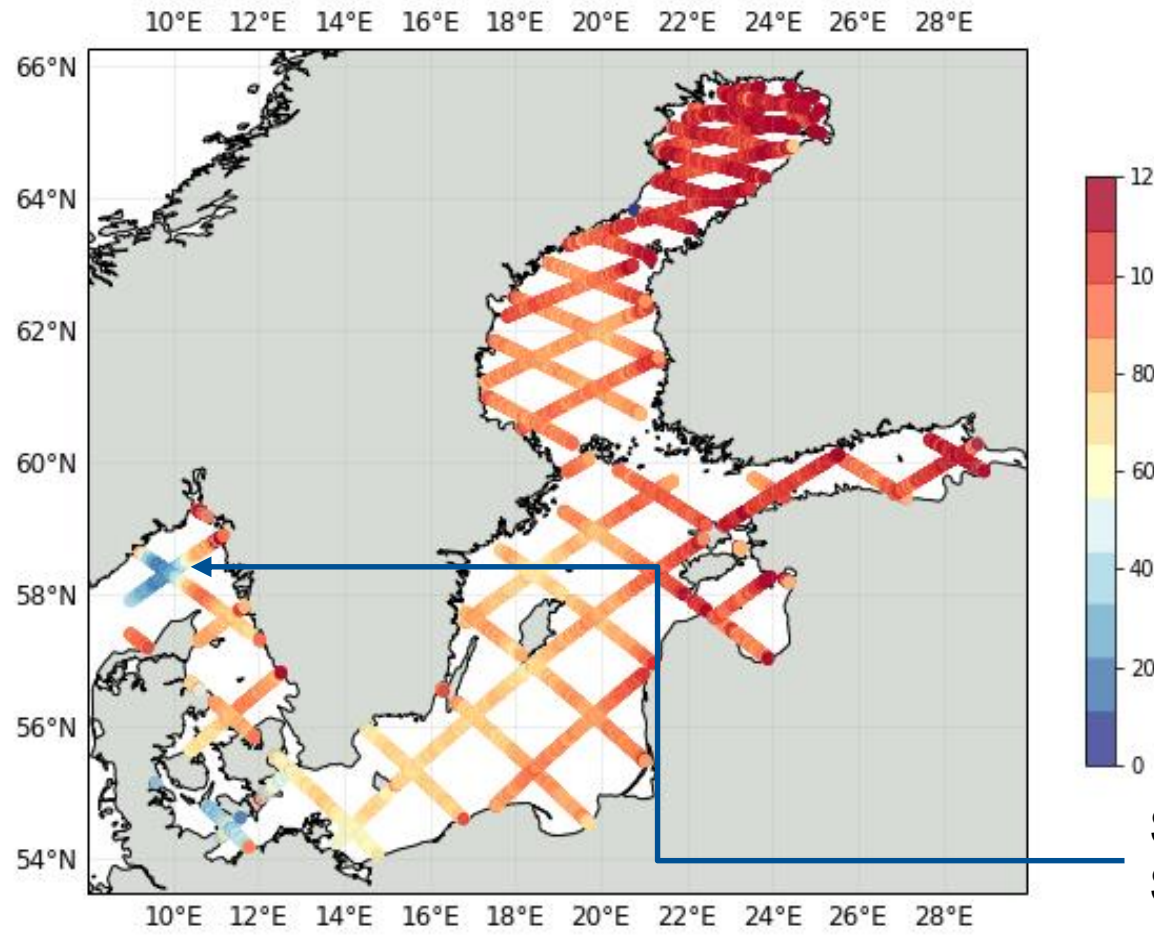

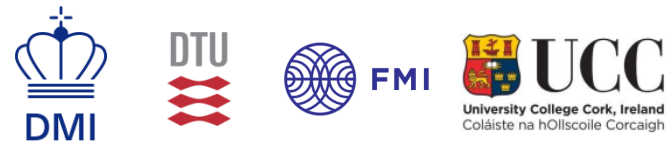

Mean annual cycle amplitude: $9 \mathrm{~cm}$ (consistent with

Stramska, M., and Chudziak, N. (2013))

Sloping annual cycle amplitude in the Skagerrak Sea [Passaro et al., 2015] 


\section{Preliminary Results: experimental high-temporal resolution gridding}

- The recent availability of several altimeters opens new possibilities for optimal interpolation every few days

- The objective is to provide a storm surge model with the best possible initial state (SSH BEFORE the surge)

- Combination of altimetry, tide gauge observations and the output of a hydrodynamic ocean model
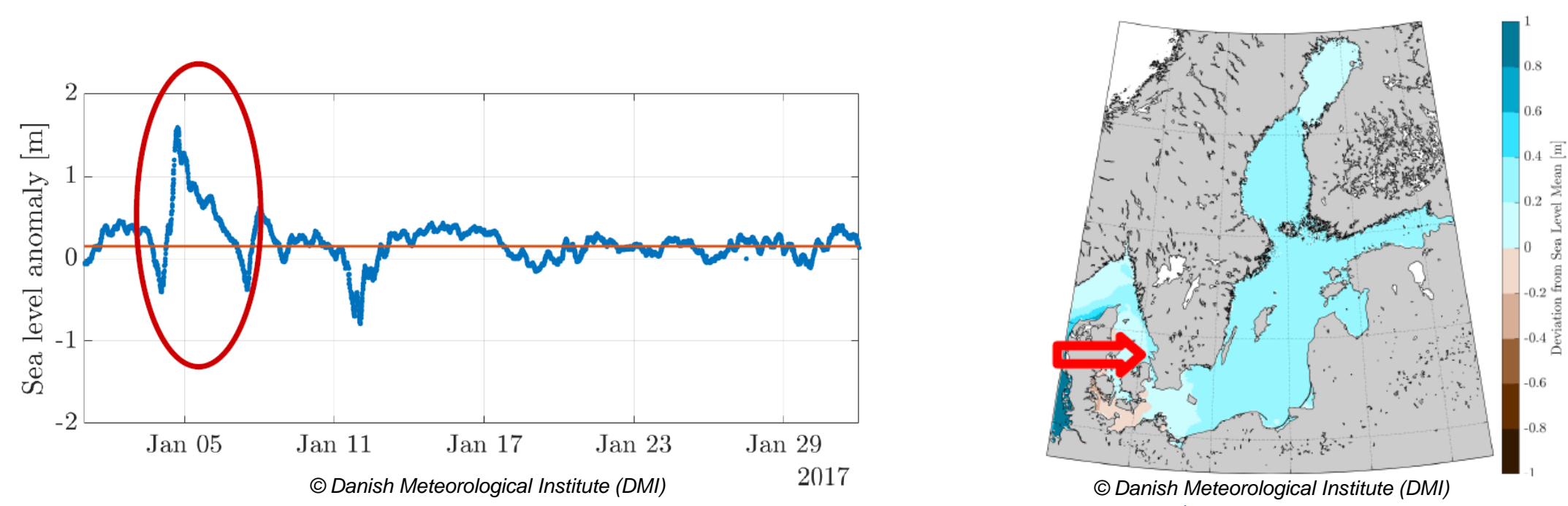


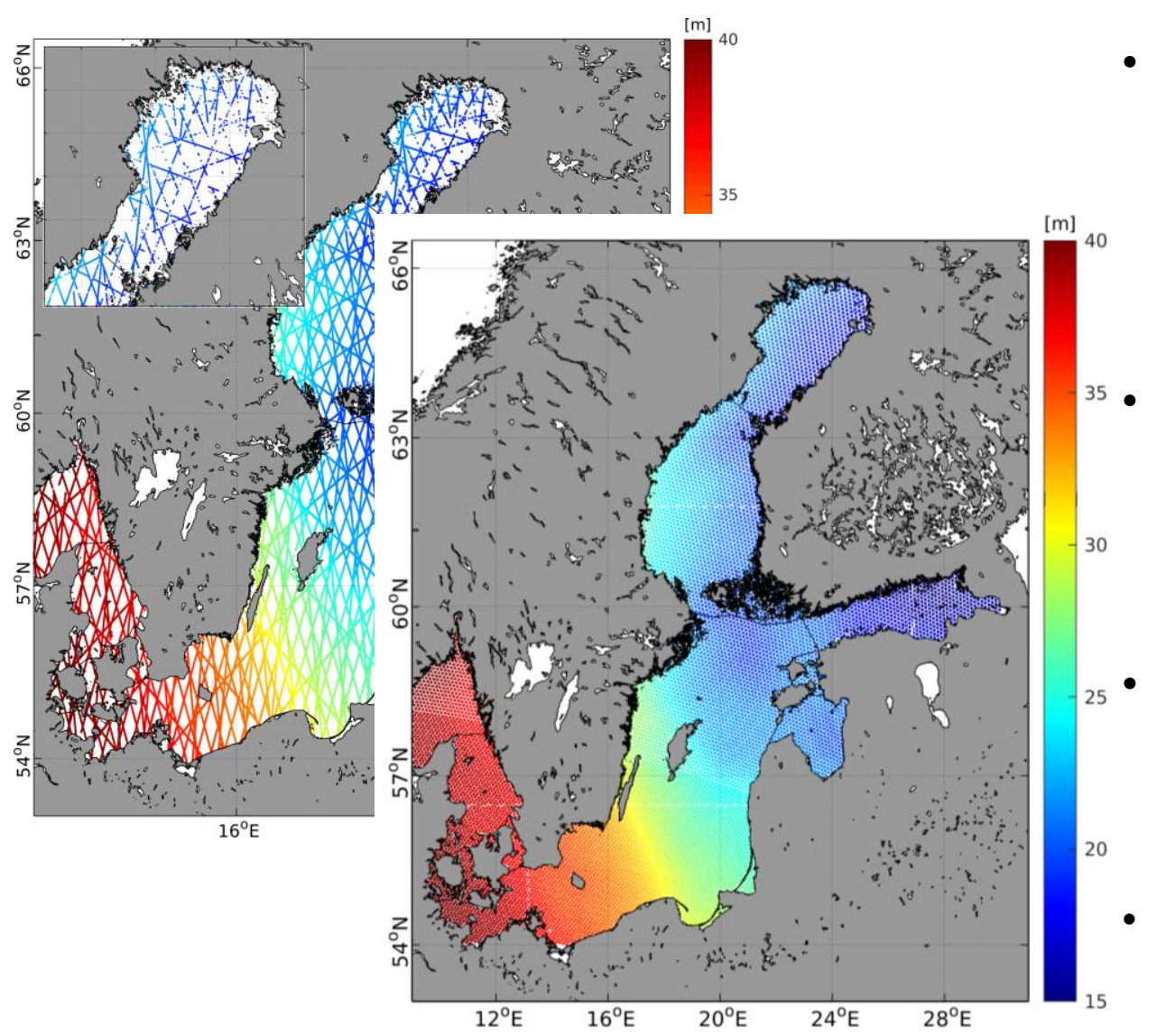

Deutsches Geodätisches Forschungsinstitut (DGFI-TUM) | Technische Universität München
- Baltic SEAL is a laboratory for high-frequency multi-mission satellite altimetry (LRM \& SAR) to produce a new Mean Sea Surface, monthly grids and high-frequent, profiled SSH products (by end of 2020)

Application of coastal dedicated retracking algorithms (ALES+) and unsupervised classification techniques to detect water opening within the sea-ice area

- Investigation of seasonal/annual sea level variability, absolute trends and identification of extreme events in the Baltic Sea

- Transfer of techniques exploited in this framework to other regions possible

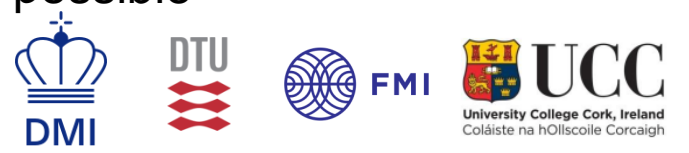


